Supporting Information

\title{
Direct coating pen ink carbon on carbonized melamine sponge as the flexible free-standing electrode
}

\author{
Jingqiu Li ${ }^{\text {a }}$, Yi Feng a,*, Mengjue Cao ${ }^{\text {a }}$, Lvye Yang a and Jianfeng Yao a,b,*
}

a Jiangsu Co-Innovation Center of Efficient Processing and Utilization of Forest Resources, College of Chemical Engineering, Nanjing Forestry University, Nanjing 210037, China

b Jiangsu Province Key Laboratory of Green Biomass-based Fuels and Chemicals, Nanjing 210037, China

*Corresponding author. E-mail: fengyi@njfu.edu.cn; jfyao@njfu.edu.cn
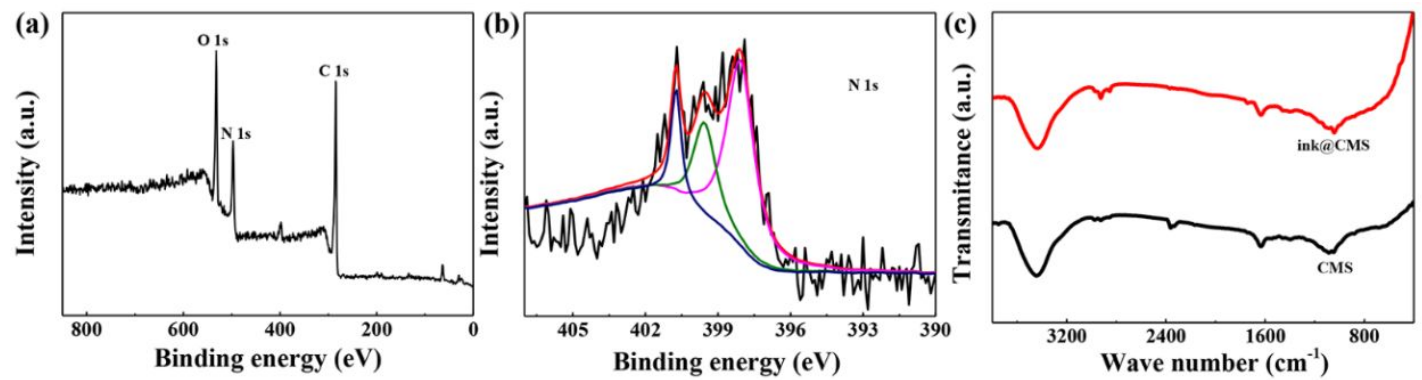

Fig. S1 XPS spectra of CMS: full scan (a) and N $1 s$ (b) and FTIR spectra of CMS and CMS@ink (c). The N 1s XPS spectrum of CMS can be deconvoluted into three peaks $(400.7,399.5$ and $398.1 \mathrm{eV})$, indicating the quaternary-N (N-Q), pyrrolic-

$\mathrm{N}(\mathrm{N}-5)$ and pyrindinic-N (N-6) respectively. N-5 and N-6 can serve as pseudocapacitance which improves the total capacitive performance while N-Q improves the electrical conductivity (Journal of Colloid and Interface Science 538 (2019) 199-208). The FTIR spectrum of CMS@ink is similar to that of CMS: the peaks at $\sim 3340,1630,1470-1560$ and $\sim 1100 \mathrm{~cm}^{-1}$ corresponding to O-H or N-H stretching vibration, $\mathrm{C}=\mathrm{C},-\mathrm{C}=\mathrm{N}$ and $\mathrm{C}-\mathrm{O}$ stretching vibration (Journal of Power Sources 2016, 336, 455-464; Journal of Materials Chemistry A 2018, 6, 17730-17739). The detection of $-\mathrm{C}=\mathrm{N}$ and $\mathrm{N}-\mathrm{H}$ peaks further proves the existence of $\mathrm{N}$ doping in the carbon framework. 

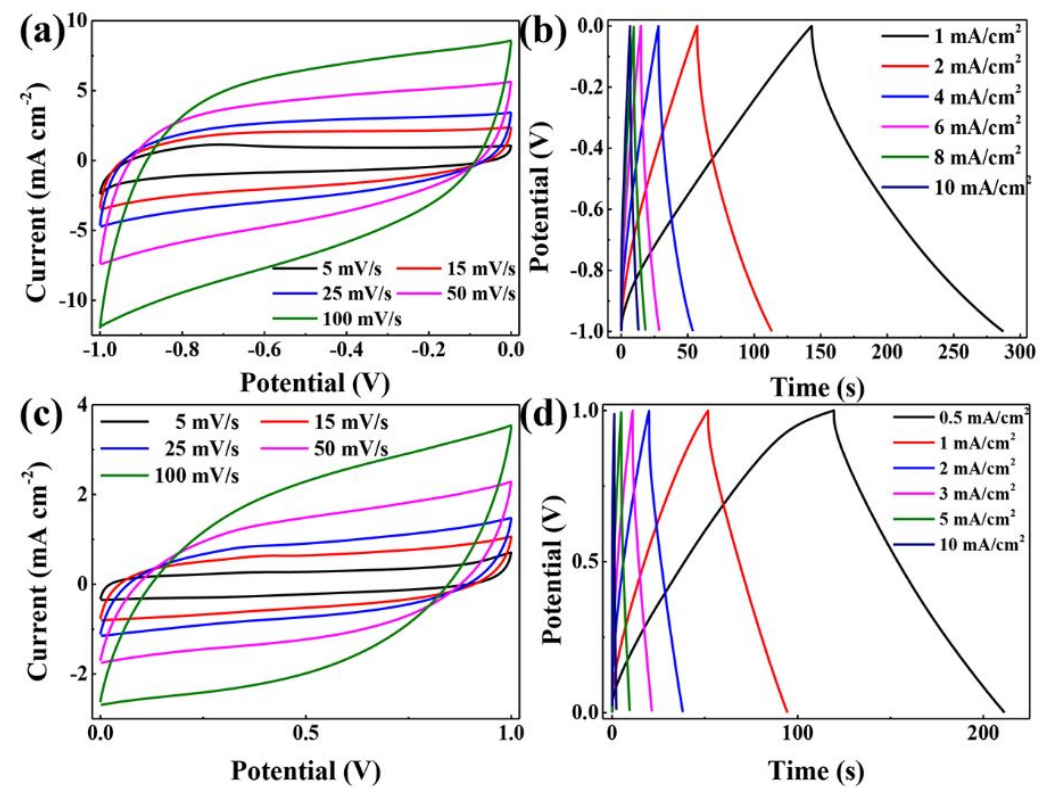

Fig. S2 Electrochemical performance of CMS in a three-electrode system $(a, b)$ and a two-electrode system (c, d) with 6.0 M KOH as the aqueous electrolyte: $\mathrm{CV}$ curves (a, c) and GCD plots $(b, d)$

(a)



(b)

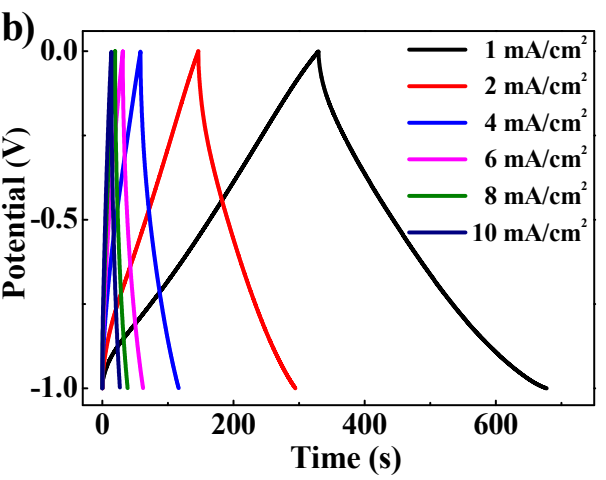

Fig. S3 CV curves of freeze-drying free-standing CMS@ink foam (a) and their

GCD plots (b) in a three-electrode system with 6.0 $\mathrm{M} \mathrm{KOH}$ as the aqueous electrolyte. 

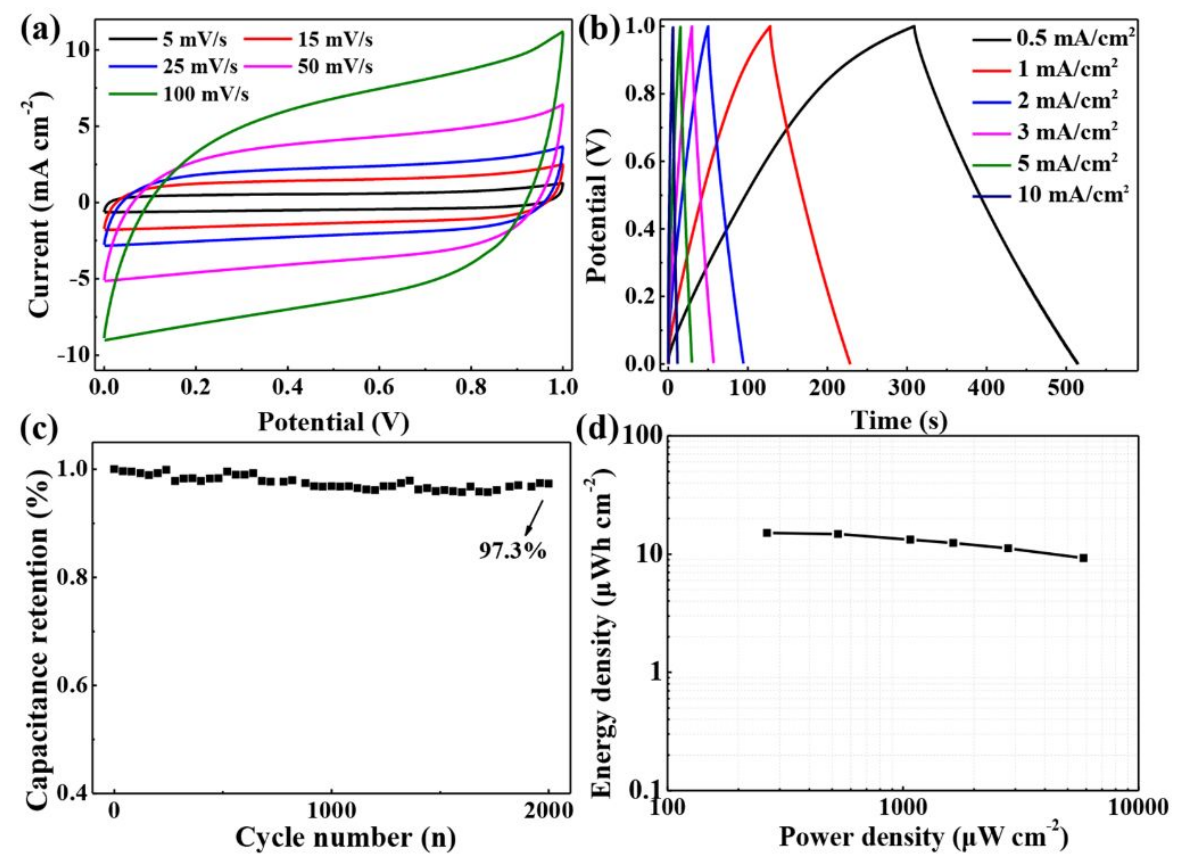

Fig. S4 The electrochemical performance of CMS@ink with polyvinyl alcohol/KOH as a solid electrolyte: CV curves (a), GCD curves (b), cycling performance (c) and the Ragone plot (d) 